\title{
BAJO RIESGO DE DÉFICIT ATENCIONAL / HIPERACTIVIDAD EN NIÑOS AYMARÁS. IMPLICANCIAS GENÉTICAS, ANTROPOLÓGICAS Y CULTURALES
}

\author{
LOW RISK OF ATTENTION DEFICIT / HYPERACTIVITY DISORDER \\ IN AYMARA CHILDREN. GENETIC, ANTHROPOLOGICAL, \\ AND CULTURAL IMPLICATIONS
}

\author{
Paula Rothhammer*, Ximena Carrasco**,****, Hugo Henríquez***, Claudia Andrade*, \\ Mario Valenzuela*****, Francisco Aboitiz* y Francisco Rothhammer***,******
}

\begin{abstract}
Se estudió la distribución de puntajes en la Escala de Conners que se utiliza para diagnosticar el trastorno por déficit atencional/ hiperactividad (TDAH) en niños del Liceo Agrícola "José Abelardo Núñez", ubicado en San Miguel de Azapa, Arica, Chile, con el propósito de desarrollar estrategias de diagnóstico precoz para prevenir este trastorno, que se asocia frecuentemente a conductas de alto riesgo, como el uso de sustancias adictivas. Las niñas y varones aymarás obtuvieron puntajes significativamente menores en comparación con los niños pertenecientes a la población no aymará. Se discuten las implicaciones genéticas, antropológicas y culturales de este hallazgo, concluyendo que posiblemente tenga como causa una interacción entre factores culturales y genéticos en concordancia con la etiología multifactorial que en general se asigna al TDAH.
\end{abstract}

Palabras claves: niños aymarás, baja prevalencia de trastornos déficit atencional/hiperactividad, etiología multifactorial.

The distribution of scores on the Conners Scale which is used to diagnose attention deficit/hyperactivity disorder (ADHD) is studied in children of the "José Abelardo Núñez" school of San Miguel de Azapa, Arica, Chile with the purpose of developing early diagnostic strategies to prevent ADHD which is frequently associated with high risk conducts, like the use of addictive substances. Aymara girls and boys rated significantly lower when compared to non-Aymara children. The genetic, anthropological and cultural implications of these findings are discussed, concluding that an interaction of cultural and genetic factors is probably responsible for our findings in agreement with the multifactorial etiology generally assigned to ADHD.

Key words: Aymara children, low prevalence of attention deficit/hyperactivity disorder, multifactorial etiology.

En términos antropológicos clásicos, la cultura es consecuencia de la herencia social (véase Linton 1965). El término cultura se usa tanto en sentido amplio, relacionándose con la herencia social de la humanidad, como en un sentido restringido, teniendo en este caso más bien relación con una modalidad particular de la herencia social que caracteriza a un determinado grupo de individuos. Indudablemente, si aceptamos estos planteamientos, la cultura en su conjunto sería la suma de un considerable número de subculturas. En este trabajo planteamos la hipótesis que estas subculturas no son solamente consecuencia de la herencia social, sino parte de la herencia biológica. Los individuos mediante sus diferentes personalidades y temperamentos interactúan fuertemente con la subcultura a que pertenecen en parte modificándola y en parte manteniéndola. Este aspecto es particularmente importante dado que personalidad y temperamento tienen una base genética.

La teoría biopsicosocial unificada de personalidad desarrollada por el genetista y psiquiatra norteamericano Robert Cloninger $(1986,1987)$ se basa en una síntesis de información de estudios de familias, estudios longitudinales y psicométricos de estructuras de personalidad, así como también en investigaciones neurofarmacológicas y neuroanatómicas, de condicionamiento y aprendizaje comportamental en el hombre y en animales.

\footnotetext{
* Departamento de Psiquiatría, Facultad de Medicina, Pontificia Universidad Católica de Chile, Santiago.

** Programa de Anatomía y Biología del Desarrollo, Facultad de Medicina, Universidad de Chile, Santiago.

*** Programa de Genética Humana, ICBM, Facultad de Medicina, Universidad de Chile, Santiago.

***** Hospital Calvo Mackenna, Santiago, Chile.

***** Departamento de Biología y Salud, Facultad de Ciencias, Universidad de Tarapacá, Casilla 6D, Arica, Chile.

****** Departamento de Antropología; Centro de Investigaciones del Hombre en el Desierto, Universidad de Tarapacá, Casilla 6D, Arica, Chile.
} 
El modelo de Cloninger se distingue de otros modelos por su integración conceptual entre la sustentación neuroanatómica y neurofísiológica de las tendencias conductuales, estilos de aprendizaje y la interacción adaptativa de dimensiones de personalidad en un esquema teórico comprensivo. La diferencia fundamental entre este modelo y otros modelos de personalidad (p. ej., Eysenk, Tellegen) es que éste postula que la estructura fenotípica observada reflejaría una estructura genética subyacente. Sin embargo, la estructura genética y fenotípica de personalidad difiere a causa de las variaciones de la interacción de las influencias ambientales y genéticas. Los procesos neurofisiológicos inducidos determinan las dimensiones básicas de personalidad, las cuales dirigen las tendencias conductuales globales y determinan la adquisición de actitudes, opiniones y creencias en una sociedad dada.

Cloninger (1987) postula la existencia de tres dimensiones de personalidad que son genéticamente independientes y que tienen patrones predecibles de interacción en sus respuestas adaptativas a los estímulos novedosos, aversivos y gratificantes. Cada sistema es complejo e implica múltiples estructuras cerebrales. Las tres dimensiones iniciales planteadas por el autor son: búsqueda de novedad (BN), evitación de daño (ED) y dependencia de la gratificación (DG). Posteriormente Cloninger (1993) amplia su teoría proponiendo un modelo de personalidad en el cual habrían cuatro dimensiones de temperamento, los ya señalados, más persistencia $(\mathrm{P})$ y tres dimensiones de carácter: cooperatividad, trascendencia y autodireccionalidad. Para Cloninger, las dimensiones de temperamento tendrían un fuerte componente genético, a diferencia del carácter que estaría más determinado por factores familiares, psicosociales y culturales.

La dimensión BN tendría una tendencia heredable hacia la actividad exploratoria frecuente y la extensa excitación en respuesta a los estímulos novedosos o gratificantes. La dimensión ED sería una tendencia heredable a responder intensamente a señales de estímulos aversivos, aprendiendo, por lo tanto, a inhibir el comportamiento para evitar el castigo, y la omisión frustrada de la recompensa no entregada. Por otra parte, se ha hipotetizado que DG sería una tendencia heredable a responder intensamente a señales de recompensa (especialmente señales verbales de aprobación social, emocionales y de apoyo) y a mantener o resistir la extinción del comportamiento que ha sido previamente asociado con recompensa o liberación del castigo.

La variación genética en cada una de las dimensiones sigue una distribución normal o de Gauss, en que la mayoría de las personas tienen valores intermedios. La dimensión ED sirve como influencia moduladora tanto en el comportamiento de búsqueda de novedad como de recompensas, llevando a la inhibición de la exploración de situaciones desconocidas y a la evitación pasiva de castigos o comportamientos no recompensados.

Como resultado de esta influencia moduladora, las influencias ambientales sobre el comportamiento de evitación de daño son compartidas entre las tres dimensiones. Específicamente, en respuesta a estímulos novedosos, la dimensión BN lleva a un comportamiento de acercamiento activo, así como la dimensión ED lleva a un comportamiento evitador pasivo o inhibido. De este modo resulta un comportamiento de equilibrio entre los acercamientos activos y la evitación pasiva.

Igualmente en respuesta a la frustración por la no recompensa, la dimensión DG predispone a un comportamiento continuado de búsqueda de recompensas, mientras que la evitación al daño predispone a la extinción del comportamiento: nuevamente el comportamiento resultante es el equilibrio sobre estas influencias de mantención y extinción. Adicionalmente DG influye en la mantención de actividades conocidas que han sido recompensadas, así como BN lleva a la iniciación de actividades desconocidas que son potencialmente gratificadoras.

La variación genética es independiente, pero al correlacionarse con la variable ambiental influyen para producir una estructura diferente de la esperada.

Los individuos que están sobre el promedio en $\mathrm{BN}$, y dentro del promedio en las otras dos dimensiones, se caracterizan por ser impulsivos, exploratorios, excitables, extravagantes, desordenados e inconstantes. Reaccionan fácilmente frente a nuevas actividades e intereses, tienden a descuidar los detalles, son distráctiles y reaccionan fácilmente frente a provocaciones peleando o huyendo. Un número importante de estos individuos, cuando niños y durante la adolescencia, sufre del llamado trastorno de déficit atencional con hiperactividad (TDHA).

En la etiología del TDHA están implicados numerosos factores biológicos. Se considera que 
es probable que exista algún tipo de disfunción cerebral a nivel del área frontal y límbica del cerebro, así como con respecto a neurotransmisores norepinefrina y dopamina. Patrones EEG anormales, como también reacciones fisiológicas disminuidas, nos sugieren una activación por debajo de lo normal. No obstante, cierta inconsistencia de los resultados impide establecer con certeza la existencia de un mal funcionamiento cerebral (WichsNelson e Israel 1999).

Las complicaciones durante el embarazo y el nacimiento se vinculan sólo de forma débil con el TDHA. La dieta no tiene más que una pequeña influencia. En contraste con esto, cada vez existe un mayor número de pruebas que apoyan una fuerte determinación genética. Alrededor del $80 \%$ de TDHA estaría genéticamente determinado (Faraone y Doyle 2001).

Se han encontrado relaciones entre el TDHA $\mathrm{y}$ factores familiares, incluyendo pobreza, estrés e interacciones familiares negativas. En la actualidad existe un gran interés por el entorno psicosocial, debido a que entornos específicos del colegio o el hogar podrían provocar, moldear y mantener conductas relacionadas con el TDHA.

El TDHA generalmente se detecta hacia los tres o cuatro años de edad y suele enviarse a los niños a recibir atención profesional durante los primeros años del colegio. A menudo se mantiene estable o se incrementa durante la niñez. Probablemente del 50 al $80 \%$ de los casos continúe durante la adolescencia y el $50 \%$ hasta la vida adulta. Hacia la adolescencia los síntomas principales tienden a reducirse, pero pueden persistir dificultades escolares, sociales y, en algunas ocasiones, conductas antisociales (Resnick 2005; Wicks-Nelson e Israel 1999). Además, se ha detectado una relación entre el TDHA y adicciones como tabaquismo, alcoholismo y abuso de sustancias (Burke et al. 2001). Un diagnóstico precoz puede permitir la intervención en etapas tempranas del desarrollo y así prevenir el incremento de conductas de alto riesgo que están asociadas al TDHA.

El presente estudio forma parte de un esfuerzo multidisciplinario que tiene, en parte, el objeto de investigar aspectos genéticos del TDHA en diferentes grupos poblacionales chilenos, incluyendo las etnias originarias, con la finalidad de aportar al diagnóstico precoz del TDHA y encauzar medidas preventivas. Cabe señalar que los protocolos del proyecto fueron aprobados por la Comisión de Bio- ética de la Facultad de Medicina de la Universidad de Chile.

\section{Sujetos y Métodos}

Se seleccionó el Liceo Agrícola "José Abelardo Núñez", ubicado en San Miguel de Azapa en la comuna de Arica, Chile, por contar entre sus alumnos con un alto porcentaje de niños pertenecientes a la etnia originaria aymará. Los profesores del colegio contestaron la encuesta abreviada de Conners (Wicks-Nelson e Israel 1999) para 396 alumnos de primero a cuarto medio. Los niños y adolescentes que puntuaron más de 15 en la encuesta fueron considerados candidatos a tener TDHA, de acuerdo a la experiencia previa ganada por nuestro grupo (Carrasco et al. 2004). Una vez aplicada la prueba de Conners, se citaron a los padres de estos alumnos a una entrevista personal con una psicóloga clínica (P.R.) y una neuróloga infantil (X.C.). Luego de firmar un consentimiento informado se obtuvieron antecedentes familiares sobre abuso de alcohol y drogas, trastornos psiquiátricos, antecedentes de embarazo y parto y TDHA. Se conversó individualmente con los profesores jefes acerca de la conducta de los niños en la sala de clase, especificando los criterios diagnósticos de DSM - IV (American Psychiatric Association 1994) para el TDHA. Finalmente, se realizó una evaluación médica del niño o adolescente por parte de la neuróloga infantil. La información obtenida fue tratada en forma absolutamente confidencial para no estigmatizar a los niños. Estos fueron luego agrupados de acuerdo a si el apellido de ambos padres era aymará (grupo 0), era aymará-no aymará (grupo 1), o bien, no aymará (grupo 2). La utilización de apellidos para determinar origen étnico ha sido ampliamente utilizado en genética de poblaciones, debido a la alta correlación que existe entre éstos y la frecuencia de genes característicos de una determinada etnia (Schull y Rothhammer 1977). Estos últimos autores proporcionan un listado completo de apellidos aymarás que sirve de referencia para la aplicación del método. Si bien es cierto que los aymarás cambian sus apellidos, el cambio siempre se realiza castellanizando los apellidos aymarás para evitar las odiosas consecuencias de la segregación, de modo que esta posible fuente de sesgo no alteraría los resultados obtenidos en niños aymarás (ver además, González y Gavilán 1990). 


\section{Resultados}

De los 396 alumnos examinados, 189 correspondieron a niñas y 207 a varones. De las niñas 91 tenían ambos padres no aymarás, 13 tenían madres aymarás y padres no aymarás, 30 tenían madres no aymarás y padres aymaras y 57 ambos padres de origen aymará. De los 207 varones 96 tenían padres de origen no aymará 18 tenían madres aymará y padres no aymarás, 36 padres aymarás y madres no aymarás y 57 ambos padres de origen aymará.

En la Tabla 1, que indica los porcentajes de niños que puntean sobre 15 en la escala de Conners, es posible observar que los porcentajes disminuyen a medida que aumenta el número de progenitores con apellidos de origen aymará. A su vez la frecuencia de varones con puntajes mayores de 15 es en general más alto que de niñas. Las diferencias son estadísticamente significativas para niñas y varones (chi cuadrado $\mathrm{p}<0.01$ ). Tendencias similares se observan al comparar en forma separada los puntajes de déficit atencional e hiperactividad (impulsividad).

\section{Discusión}

Nuestros resultados indican que los niños de origen aymará presentan menores puntajes en la Escala de Conners que sus compañeros pertenecientes a la población no aymará del Valle de Azapa. Estos resultados son válidos tanto para niñas como varones exhibiendo los varones valores algo más altos siguiendo una tendencia que es observable a nivel mundial (Anderson et al. 1987, Baumgaertel et al 1995; Gómez et al. 1999; Rohde et al. 1999; Tahir et al. 2000; Wilens et al. 2002; Wolraich et al. 1996). Nuestros resultados se ven reforzados por las entrevistas realizadas a los profesores que indican que los niños de origen ayma-

Tabla 1. Frecuencia relativa de niños con puntaje superior a 15 en la escala de Conners.

Relative frequencies of children that scored higher than 15 in the Conner's scale.

\begin{tabular}{|c|c|c|c|c|}
\hline \multirow[t]{2}{*}{ Género } & \multirow[t]{2}{*}{$\mathrm{N}$} & \multicolumn{3}{|c|}{ Número de Apellidos Aymarás } \\
\hline & & 0 & 1 & 2 \\
\hline Niñas & 189 & $16 / 91 \quad(17,6 \%)$ & $2 / 43(4,7 \%)$ & $2 / 55(3,6 \%)$ \\
\hline Varones & 207 & $23 / 96 \quad(23,9 \%)$ & $9 / 54(16,7 \%)$ & $2 / 57(3,5 \%)$ \\
\hline Total & 396 & $39 / 187(20,8 \%)$ & $11 / 97(11,3 \%)$ & $4 / 112(3,6 \%)$ \\
\hline
\end{tabular}

rá tienden a ser más ordenados, ponen mayor atención en clase y no molestan a sus compañeros.

El comportamiento de los niños aymarás puede tener un origen cultural en el sentido que existen normas ancestrales que son reforzadas por la comunidad étnica frente a la sociedad nacional, que aunque no son comúnmente valoradas en la población no aymará del Valle de Azapa.

Otra causa del comportamiento de los niños de esta etnia podría estar relacionada con la composición genética de la misma. En este contexto cabe mencionar que se han realizado numerosos estudios que indican la existencia de una asociación entre alelos de genes que determinan la morfología molecular de los receptores de dopamina y serotonina, como también del transportador (DAT) con puntajes altos en la Escala de Conners. Estos polimorfismos de genes relacionados con neurotransmisores han sido vinculados al TDHA en varias poblaciones (Vieyra et al. 2003).

Las hipótesis cultural y genética no son mutuamente excluyentes, de modo que los resultados obtenidos puedan deberse a una interacción de ambos factores en el sentido que una determinada composición genética determine el desarrollo de patrones conductuales que, a su vez, influyen en el devenir cultural, que modula el comportamiento individual de los miembros de una comunidad.

Si definimos personalidad como el conjunto de rasgos psíquicos del individuo, es decir, la suma total de sus facultades racionales, percepciones, ideas, hábitos y reacciones emotivas condicionadas, que conforman una configuración cuyas partes funcionan en constante relación mutua, no podemos dejar de reconocer que existe una estrecha relación entre esta configuración y la cultura de la sociedad a la que el individuo pertenece. La cultura existe en las mentes de los individuos que componen una sociedad y deriva sus cualidades de sus respectivas personalidades. A la inversa, la personalidad de los individuos que componen una sociedad se desarrolla en constante asociación con su cultura. Las personalidades afectan a la cultura y la cultura a las personalidades dentro de un esquema dinámico. En consecuencia, las culturas no son estáticas, y por lo tanto, el corte transversal del continuo cultural estudiado en un determinado momento por un antropólogo tendría, por ejemplo, la misma relación con el conjunto que la escena de una obra teatral con la obra completa (Linton 1965).

Hemos mencionado que el TDHA se asocia a conductas de alto riesgo, a mal rendimiento esco- 
lar y al desarrollo de adicciones como el tabaquismo, alcoholismo y drogadicción. Resulta evidente que la posibilidad de realizar un diagnóstico precoz de este trastorno en los niños puede ser un factor de prevención importante. Los conocimientos que se obtengan sobre la etiología del TDAH, tomando en consideración tanto los determinantes antropológicos culturales presentes en nuestra población multiétnica como su composición genética, estudiada utilizando las herramientas actuales de la biología molecular, son importantes para lograr este objetivo.

Agradecimientos: A la Directora señora Margarita Martínez Vega y a los profesores del Liceo Agrícola "José Abelardo Núñez" por su amable coloboración con esta investigación, como también el financiamiento del Proyecto Fondecyt $\mathrm{N}^{\circ} 1050721$. A los evaluadores anónimos que con sus comentarios desinteresados contribuyeron a mejorar sustancialmente este trabajo.

\section{Referencias Citadas}

American Psychiatric Association 1994 DSM-IV: Diagnostic and Statistical Manual of Mental Disorders. $4^{\text {th }}$ ed. American Psychiatric Association, Washington, D.C.

Anderson, J.C., S. Williams, R. McGee y P.A. Silva 1987 DSM-III disorders in preadolescent children. Prevalence in a large sample from the general populations. Arch Gen Psychiatry 44:69-76.

Baumgaertel, A., M.L. Wolraich y M. Dietrich 1995 Comparison of diagnostic criteria for attention deficit disorders in a German elementary school sample. J. Am. Acad. Child. Adolesc. Psychiatry 34:629-638.

Burke, J., R. Loeber y B. Lahey

2001 Which aspects of ADHD are associated with tobacco use in early adolescence. J. Child Psychol. Psychiat 42:493-502.

Carrasco, X., P. Rothhammer, M. Moraga, H. Henríquez, F. Aboitiz y F. Rothhammer

2004 Presencia de los alelos DRD4/7R y DAT/10R en miembros de familias chilenas con síndrome de déficit atencional con hiperactividad. Rev. Med. Chile 132:1047-1052.

Cloninger, C.R.

1986 A unified biosocial theory of personality and its role in the development of anxiety states. Psychiatr Dev. 4:167-226. 1987 Neurogenetic adaptive mechanisms in alcoholism. Science 236:410-416.

Cloninger, C.R., D.M. Swakic y T.R. Przybeck

1993 A psychological model of temperament and character. Arch Gen Psychiatry 50:991-999.

Faraone, S.V. y A.E. Doyle

2001 The nature and heritability of attention-deficit/hyperactivity disorder. Child Adolesc Phychiatry Clin N Am 10:299-316.

Gómez R., J. Harvey, C. Quick, I. Scharer y G. Harris 1999 DSM-IV AD/HD: confirmatory factor models, prevalence, and gender and age differences based on parent and teacher ratings of Australian primary school children. J. Child Psychol Psychiatry 40:265-274.

González, H. y V. Gavilán 1990 Cultura e identidad étnica entre los aymaras chilenos. Chungara 24/25:145-158.
Linton, R.

1965 Estudio del Hombre. 8므 Edición, Fondo Cultura Económico, México.

Resnick, R. J.

2005 Attention deficit hyperactivity disorder in teens and adults: They don't all outgrow it. J Clin Psychol. 61:529-33.

Rohde, L.A., J. Biederman, E.A. Busnello, H. Zimmermann, M. Schmitz, S. Martins y S. Tramontina

1999 ADHD in a school sample of Brazilian adolescents: a study of prevalence, comorbid conditions and imparirments. J Am Acad Child Adolesc Psychiatry 38:716-722.

Schull, W.J. y F. Rothhammer

1977 A Multinational Andean Genetic and Health Program: Rationales and Desing for a Study of Adaptation to Hypoxia of Altitude. En Genetic and Non-Genetic Components of Physiological variability, editado por J. S. Wiener, SSHB Pub. 17 pp. 139-169, London.

Tahir, E., Y. Yazgan, B. Cirakoglu, F. Ozbay, I. Waldman y P.J. Asherson

2000 Association and linkage of DRD4 and DRD5 with attention deficit hyperactivity disorder (ADHD) in a sample of Turkish children. Mol Psychiatry 5:396-404.

Vieyra, G., M. Moraga, H. Henríquez, F. Aboitiz y F. Rothhammer

2003 Distribution of DRD4 and DAT1 alleles from dopaminergic system in a mixed Chilean population. Rev Med Chil. 131:135-143.

Wichs-Nelson, R. y A. Israel

1999 Psicopatología del Niño y del Adolescente. $3^{\mathrm{a}}$ Ed. Prentice Hall, Madrid.

Wilens, T.E., J. Biederman y T.J. Spencer

2002 Attention deficit/hyperactivity disorder across the lifespan. Апnu Rev Med 53:113-131.

Wolraich, M.L., J.N. Hannah, T.Y. Pinnock, A. Baumgaertel y J. Brown

1996 Comparison of diagnostic criteria for attention-deficit hyperactivity disorder in a county-wide sample. J Am Acad Child Adolesc Psychiatry 35:319-324. 